\title{
$\infty$
}

\section{Development of a Piezoelectric Ultrasonic Tooth-whitening Apparatus}

\author{
Young-Jin Lee ${ }^{\dagger}$ and Jong-Hoo Paik \\ Korea Institute of Ceramic Engineering and Technology, Seoul 153-801, Korea \\ Jeong-Bae Lee and Seung-Jae Choi
Dmetec Co., Ltd., Bucheon 402-603, Korea
}

Received June 24, 2013; Revised August 2, 2013; Accepted September 3, 2013

\begin{abstract}
The aim of this study was to develop an ultrasonic tooth-whitening apparatus using piezoelectric transducers, which enhance bleaching efficiency by applying ultrasound, while performing a teeth whitening procedure. For this purpose, an ultrasonic transducer was designed and manufactured, and the effects of reduction in the whitening material's concentration and in the whitening treatment time through using the ultrasound cavitation phenomenon were confirmed. Also, the validity of this study was investigated by comparing the whitening performance with a commercialized optical whitener, through color comparison. The results revealed that the ultrasound whitener produced color values that were enhanced by as much as double that of the conventional LED light whitening method. Even when the operational time was reduced by half, the ultrasound method showed superior performance by over $54 \%$ compared to the conventional light whitener, revealing that the ultrasound method showed a remarkable treatment reduction effect.
\end{abstract}

Keywords: Ultrasonic, Piezoelectric, Tooth-whitening, Dental bleaching

\section{INTRODUCTION}

The color of teeth is determined by internal color change and external coloring. Internal color change is related to the light scattering and absorption of the enamel and dentin. In the case of external coloring, the main cause of teeth coloring is the absorption of staining substances by the enamel surface from drinking beverages and smoking which occurs in everyday life. With the recent trend of emphasizing beauty, the number of people undergoing whitening is increasing including patients who had difficulty conducting home bleaching, and people seeking a short-term shade improvement. Also, as whitening is an advanced technology industry, there is a high chance that the

${ }^{\dagger}$ Author to whom all correspondence should be addressed: E-mail: yjlee@kicet.re.kr

\section{Copyright $@ 2013$ KIEEME. All rights reserved.}

whitening markets will continuously grow, both quantitatively and qualitatively, with increasing national income [1,2].

Teeth whitening involves the main ingredients hydrogen peroxide(HDP) and carbamide peroxide(CBP) in teeth whiteners, which are broken down inside the oral cavity by heat or light to release oxygen, and released oxygen enters the enamel and dentin to bleach the colored material, so that the teeth become white and bright. The whiteners HDP and CBP are used in various concentrations from $10 \%$ to $35 \%$, and although their whitening performance is greater with higher concentrations. It has been reported that high concentrations lead to side effects, such as discomfort of the gingival tissue and sensitivity to cold stimulation $[3,4]$. The method simultaneously using light and heat results in temperature increases of the teeth, causing hypersensitivity due to vasodilation within the dental pulp. At present, whiteners using light irradiation are mainly being commercialized [5-7].

Ultrasound is used in everyday life and in various industries, such as precision processing, machinery, semiconductor, and 
vitreous flow; ultrasound medical diagnosis; and ultrasonic cleaning [8-10]. Among these, the cavitation phenomenon using ultrasound occurs as bubbles are formed about foreign substances in the medium, the bubbles repeatedly forming and dissipating. Then, the bubbles grow until they become larger than a certain size, and contract and rupture all at once; and the produced shockwave creates high pressure and temperature. This effect accelerates physical and chemical reactions. The high pressure and temperature from cavitation causes physical damage, breaks down the binding of cells, and produces free radicals that can react with microbial cells, making sterilization possible.

The aim of this study is to develop an ultrasonic tooth-whitening apparatus using piezoelectric transducers, which enhance bleaching efficiency by applying ultrasound, in comparison with the commercialized optical whitening method. Increasing whitening efficiency means not only reduction of the procedure time, but also reduction of the toxic whitening materials.

For this purpose, an ultrasonic whitening apparatus comprising 3 unit piezoelectric transducers was designed and manufactured, and the whitener concentration reduction and whitening treatment time reduction effects using the ultrasound cavitation phenomenon were confirmed. Also, the validity of this study was investigated, by comparing the whitening performances with acommercialized optical whitener through color comparison.

\section{EXPERIMENTS}

\subsection{Ultrasonic transducer design}

To design the ultrasonic whitening apparatus using piezoelectric transducers for this purpose, the commercial finite element analysis program ANSYS 10.0 was used for the structural design and analysis of the behavior of the transducer. An ultrasonic frequency of 1 2 MHz of small wavelength was chosen to effectively transfer the hydrogen peroxide whitening material to the teeth. Also, the whitening apparatus consisted of 3 piezoelectric unit transducers configured at 45 degree in the form of a mouthpiece jig, as shown in Fig. 1, to secure a uniform ultrasound pressure distribution of the whitening medium.

The front surface of the piezoelectric element, composed of electrodes and an oxidation barrier layer, directly contacts the whitening medium, and a silicon absorber layer is placed at the side surface to act as a support of the vibration and to block whitening medium passing into the whitening apparatus. A radial type PZT is used as piezoelectric material, having a shape of 20 $\mathrm{mm}$ diameter and $1.3 \mathrm{~mm}$ thickness, which results in a resonant frequency of $1.68 \mathrm{MHz}$ of thickness vibration mode. An air gap is placed on the backside of the PZT so that the maximum vibration energy can be delivered to the opposite side.

The properties used in the design are shown in Table 1, and through modal and harmonic analysis, an ultrasonic transducer with a resonant frequency of 1.6 1.8 MHz and a whitening apparatus were designed.

\subsection{Whitening experiment}

Ceramic HA(Hydroxyapatite, TAIHEI CHEMICAL, Japan), which has a crystallographic and chemical composition similar to that of human bones was used as the material substituting for teeth, to investigate a whitening performance. It was sintered for 2 hours, by raising the temperature to $1,350^{\circ} \mathrm{C}$ at a rate of $5^{\circ} \mathrm{C} /$ min. Artificial teeth specimens of disk shape of $14 \mathrm{~mm}$ diameter and $2 \mathrm{~mm}$ thickness were fabricated. The prepared specimens were mirror polished, and stained for one week at room tem-
Table 1. Material properties used forANSYS analysis.

\begin{tabular}{cccc}
\hline Material & $\begin{array}{c}\text { Density } \\
\left(\mathrm{kg} / \mathrm{m}^{3}\right)\end{array}$ & $\begin{array}{c}\text { Modulus of } \\
\text { Elasticity }(\mathrm{GPa})\end{array}$ & Poisson's ratio \\
\hline \hline PZT & 7,750 & 60 & 0.31 \\
Aluminum & 2,700 & 70 & 0.33 \\
Silicone rubber & 1,400 & 0.01 & 0.50 \\
Air & 1.21 & 0.14 & 0.30 \\
\hline
\end{tabular}
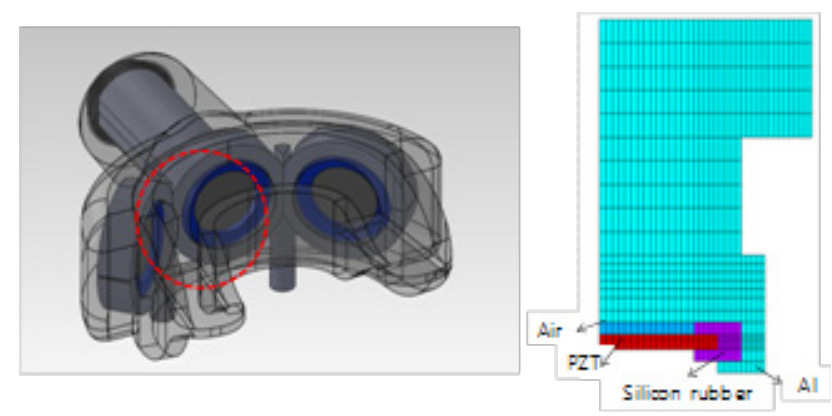

Fig. 1. Modeling of the ultrasonic tooth-whitening apparatus, and cross sectional diagram of a unit piezoelectric transducer.

perature using a solution of instant coffee, black tea and wine. Figure 2 shows uncolored and colored HA specimens.

The whitening assessment involved 10 samples for each experimental condition to obtain the average value, and was conducted by immersing the colored tooth replacement HA specimens in $15 \%$ hydrogen peroxide at room temperature, and incrementally increasing the exposure time to ultrasonic vibration or an LED light source, from 20 minutes to 1 hour, to measure the $\mathrm{pH}$ and content changes [11]. Figure 3(a) shows a whitener using a conventional LED light source(Aladdin, DXM.CO.LTD, Korea), and Fig. 3(b) shows the manufactured ultrasonic transducer. Evaluation of the degree of whitening was performed using a spectrophotometer(CN-700d, KONICA MINOLTA, JAPAN), and the differences in perceived color space $A$ before whitening (CIE 1976, Color space, $\left.L^{*}, a^{*}, b^{*}\right)$ and after whitening $A^{\prime}\left(L^{*^{\prime}}, a^{*^{\prime}}, b^{* \prime}\right)$, were compared [12].

\subsection{Ultrasound driving circuit}

In real application, movement of the resonant frequency of the ultrasonic transducer according to the amount, concentration, and temperature of the hydrogen peroxide, as well as the teeth bite shape, becomes a matter of concern. Therefore, by varying the frequency through a voltage controlled oscillator(VCO) circuit, and searching for the frequency when the impedance of the piezoelectric transducer was the lowest, the resonant frequency can be tracked through the driving algorithm shown in Fig. 4.

Since the resonance frequency of the selected piezoelectric transducer is in the range of about 1 2 MHz, a frequency generation module was designed by using a PWM(pulse width modulation) controller. When power is supplied to the teeth whitener, the microprocessor(hereafter MCU) delivers the current status to the display module, and according to the PWM duty, the frequency generation module generates a frequency of $1.3 \sim 1.9 \mathrm{MHz}$ passing through the power switching and matching circuit to be finally delivered to the piezoelectric transducer. The impedance list is delivered to the MCU, and the MCU searches the resonant frequency of the piezoelectric transducer. Then, the PWM duty corresponding to the searched resonant frequency is delivered 

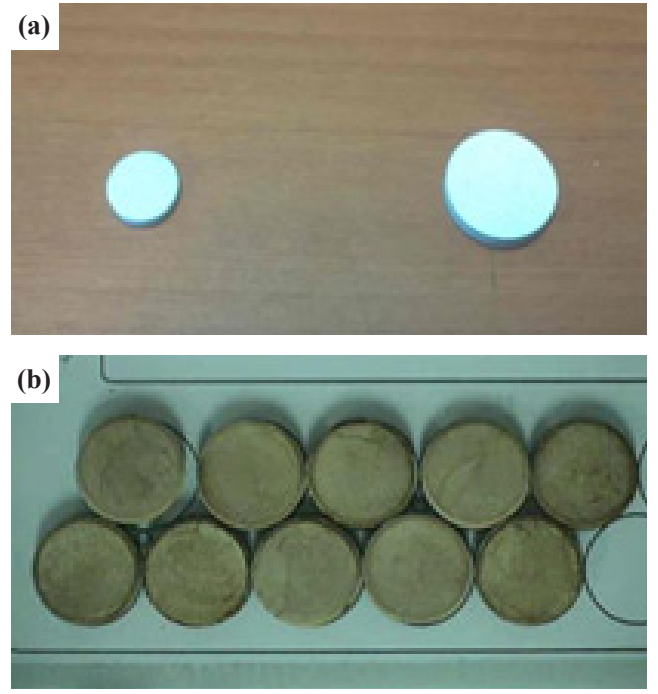

Fig. 2. The colored HA specimen.
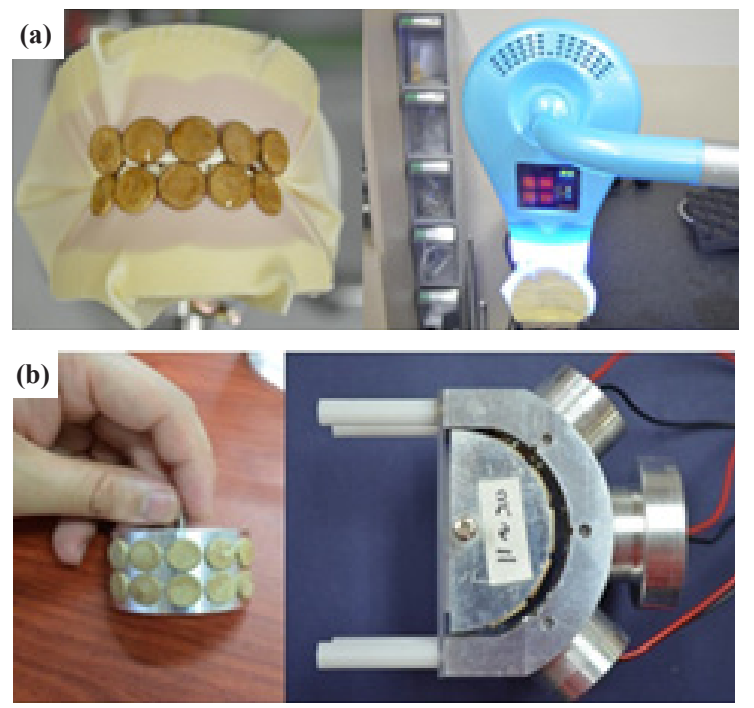

Fig. 3. Experimental setup of the (a) optical (LED) whitening and (b) ultrasonic whitening.

to the frequency generation module, and the piezoelectric transducer can be operated at the resonant frequency. The constantcurrent circuit was composed so that user defined output is maintained at a constant level.

\section{RESULTS AND DISCUSSION}

\subsection{Ultrasonic transducer \& apparatus}

Verification of the validity of the structure is carried out through finite element analysis. Figure 5(a) is a meshed diagram of the unit ultrasonic transducer consisting of 1,704 nodes and 1,610 elements. Results of the harmonic analysis and fabricated transducer's impedance are shown in Fig. 5(b). The frequency response characteristic of the transducer is checked with an impedance analyzer, HP4192A. Some difference of the response is believed to happen because of the difference of the material properties, such as parasitic capacitance and inductance, and

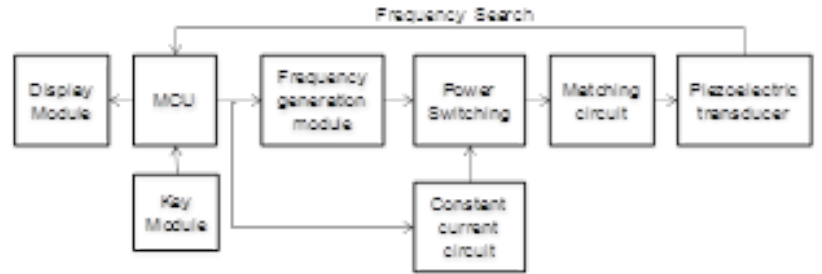

Fig. 4. Resonant frequency tracking algorithm for the ultrasonic transducer

(a)
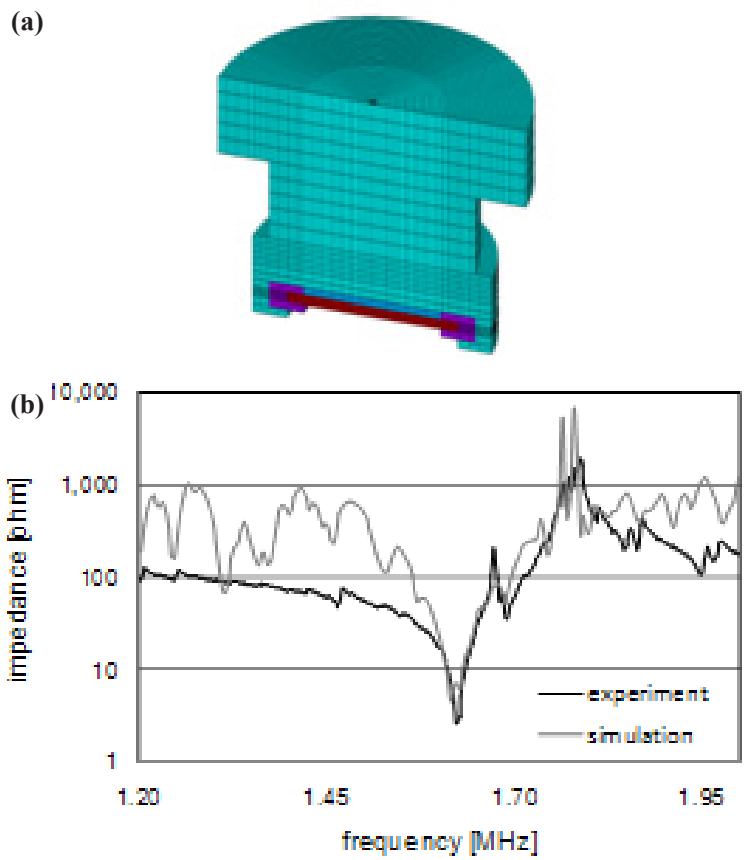

Fig. 5. Unit piezoelectric transducer (a) half model diagram and (b) impedance plots of the simulation and actual measurement.

also the difference of loss coefficients used in ANSYS analysis. However, it shows good agreement between the two results over the resonant frequency of $1.68 \mathrm{MHz}$. Figure 6(a) shows ameshed diagram of the ultrasonic transducers, with 3 cells a rranged at 45 degree. The results show that the generated ultrasound is evenly radiated in the medium, and the average sound pressure was about 8,387 Pa, due to the ultrasound generated at the front surfaces where the teeth were placed.

Based on these results, a prototype of the ultrasonic whitener was manufactured, and measurement of the resonant frequency was found to be $1.68 \mathrm{MHz}$, which is in good agreement with the harmonic analysis result.

\subsection{Whitening evaluation}

Even though the ultrasonic cavitation is affected by the medium concentration, $\mathrm{pH}$, temperature, pressure, time and frequency, the ultrasound radiation may cause changes in the experimental conditions, such as the medium concentration, temperature and $\mathrm{pH}$. Therefore, prior to the whitening assessment, the concentration and $\mathrm{pH}$ changes of the HDP were investigated according to the ultrasound and LED light exposure, respectively.

After exposing specimens to each whitening condition for an increasing experiment time from 20 minutes to 1 hour, it was 

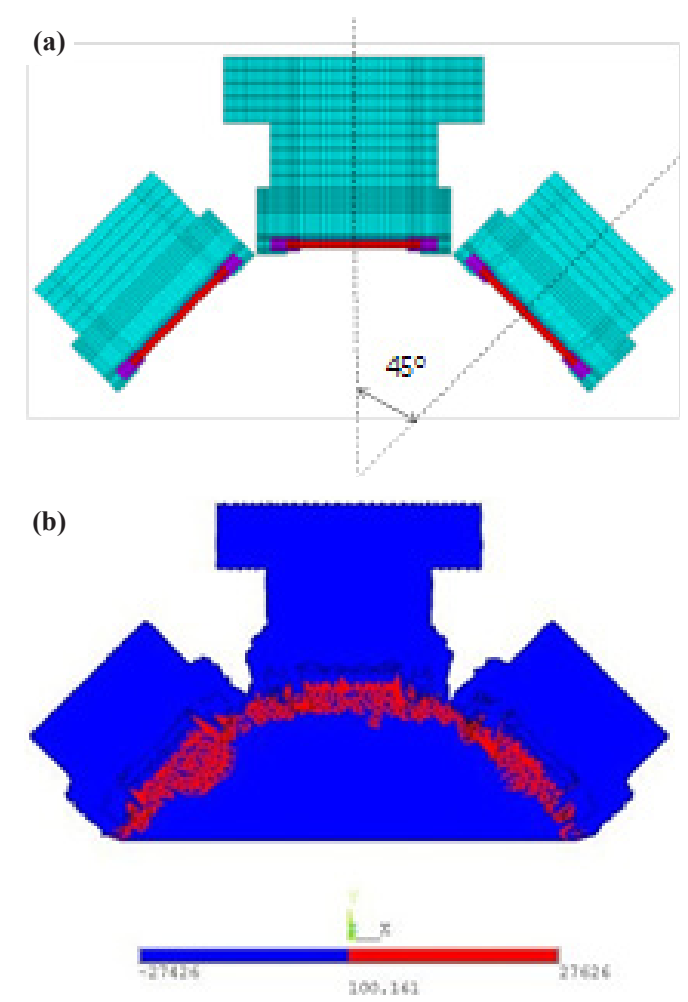

Fig. 6. Ultrasonic transducers composed of 3 unit cells (a) modeling diagram and (b) simulated ultrasound pressure distribution.

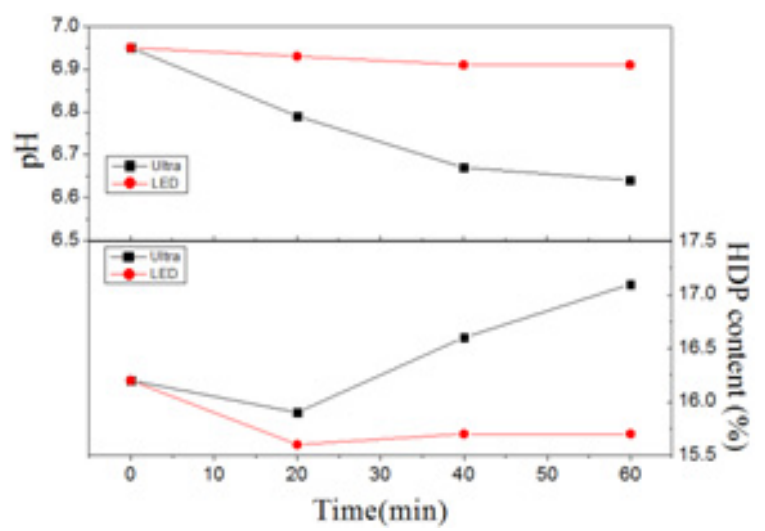

Fig. 7. The HDP content and $\mathrm{pH}$ changes according to the test time.

found that the HDP content and $\mathrm{pH}$ change from the ultrasound and light were as shown in Fig. 7. In the case of the ultrasound method, an increase in temperature of about $2 \sim 5$ degrees Celsius occurred, due to the vibration friction by the piezoelectric element, which led to relatively larger changes in the $\mathrm{pH}$ and HDP concentration compared to the LED method. However, an overall change within $5 \%$ was found, which led to the conclusion that the experimental condition changes due to ultrasound and the LED light source were insignificant. The electric power applied to the piezoelectric transducer was $10 \mathrm{~W}$.

The color of the HA specimens were measured using a spectrophotometer before and after each whitening process, for 3 intervals of 20 minutes to 1 hour under equal condition. As seen in Fig. 8, the ultrasonic whitening method produces superior whitening improvement compared to the conventional LED method, and the chrominance measurement results shown in Fig. 9 reveal
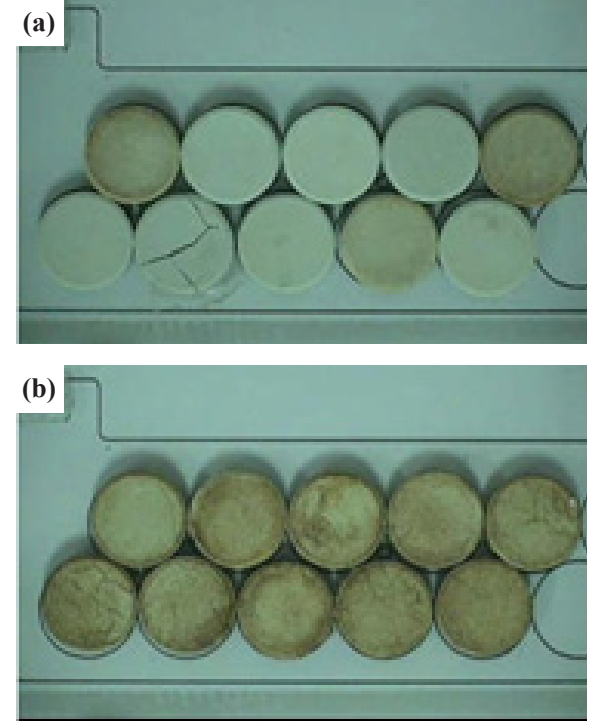

Fig. 8. HA samples after the ultrasonic and LED whitening processes (a) after ultrasonic process and (b) after LED process.

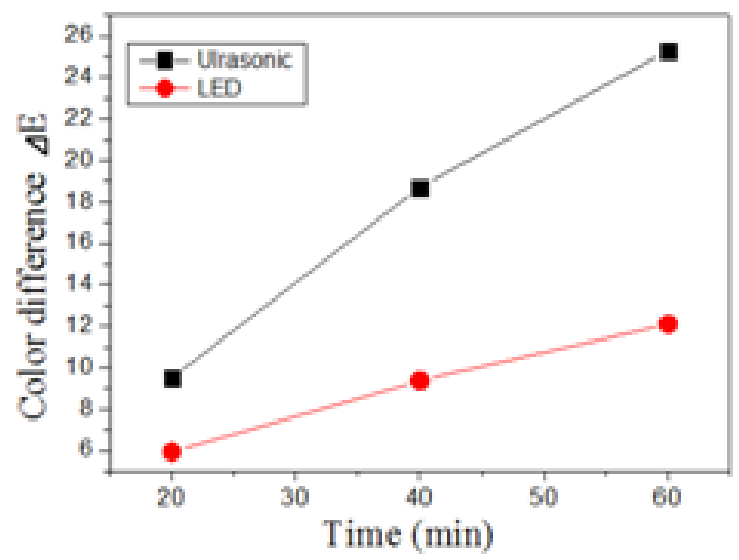

Fig. 9. Comparison of the $\Delta \mathrm{E}$ between the ultrasonic and LED whitening methods (60 minutes).

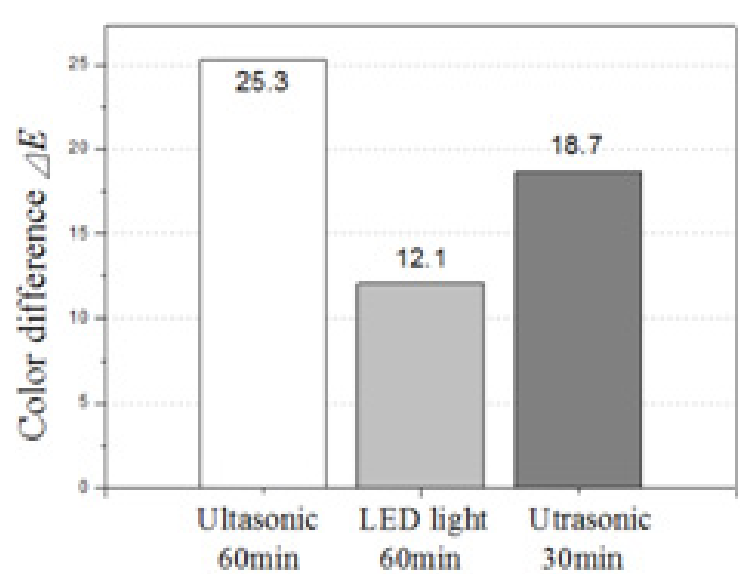

Fig. 10. Comparison of the $\Delta \mathrm{E}$ between the ultrasonic and conventional LED whitening methods. 


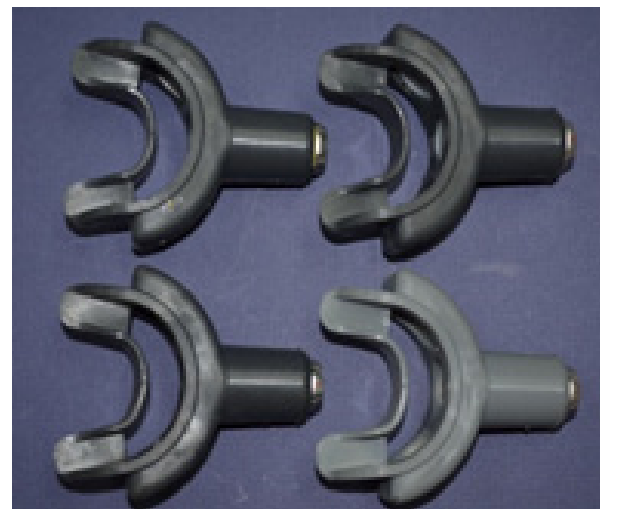

Fig. 11. The prototype sample of the ultrasonic tooth-whitening apparatus.

that there was an improvement of approximately double from the values of 12.1 and 25.3, respectively.

Figure 10 shows the result of chrominance comparison between 1 hour process and reduced time of 30 minutes. The reduced 30 minutes process showed an approximately $54.3 \%$ improved result, compared to that of the 1 hour LED method. As a result, it was determined that the use of ultrasound effectively led to a reduction of whitener treatment time.

Figure 11 shows the prototype sample of the piezoelectric ultrasound whitener, in which 3 piezoelectric transducers are attached to the mouthpiece shape jig, arranged at 45 degree.

\section{CONCLUSIONS}

In this study, an ultrasonic tooth-whitening apparatus using piezoelectrict ransducers was developed and evaluated, which enhances bleaching efficiency by applying ultrasound while the teeth whitening procedure is being performed. For this purpose, an ultrasonic transducer was designed and manufactured, and the whitening material's concentration reduction and whitening treatment time reduction effects using the ultrasound cavitation phenomenon were confirmed. Also, the validity of this study was investigated by comparing the whitening performance with a commercialized optical whitener through color comparison. The results revealed that the ultrasound whitener produced color values that were enhanced by as much as double that of the con- ventional LED light whitening method. Even when the operation time was reduced by half, the ultrasound method showed superior performance by over $54 \%$, compared to the conventional light whitener, revealing that the ultrasound method showed a remarkable treatment reduction effect.

\section{ACKNOWLEDGMENTS}

This work was supported by anEnergy Efficiency \& Resourcesof the Korea Institute of Energy Technology Evaluation and Planning(KETEP) grant, funded by the Korea government Ministry of Knowledge Economy (No. 20112020100040).

\section{REFERENCES}

[1] P. W. Kihn, Dent. Clin. Nor. Am. 51, 319 (2007) [DOI: http:// dx.doi.org/10.1016/j.cden.2006.12.001].

[2] S. Y. Kim, J. U. Park, C. H. Kim and S. E. Yang, J. Kor. Acad. Cons. Dent. 35, 40 (2010) [DOI: http://dx.doi.org/10.5395/JKACD. 2010.35.1.040].

[3] R. R. Seghi and I. Denry, J. Dental Research 71, 1340 (1992) [DOI: http://dx.doi.org/10.1177/00220345920710061201].

[4] U. Oltu and S. Gurgan, J. Oral Rehabil. 27, 332 (2000) [DOI: http://dx.doi.org/10.1046/j.1365-2842.2000.00510.x].

[5] Y. S. Noh et al., J. Kor. Acad. Cons. Dent. 36, 306 (2011) [DOI: http://dx.doi.org/10.5395/JKACD.2011.36.4.306].

[6] J. C. Ontiveros and R. D.Paravina, J. Dent. 37, 840 (2009) [DOI: http://dx.doi.org/10.1016/j.jdent.2009.06.015].

[7] J. H. Park, H. J. Shin, D. Y. Park, J. W. Kim and K. M. Cho, J. Kor. Acad. Cons. Dent. 34, 95 (2009) [DOI: http://dx.doi.org/10.5395 /JKACD.2009.34.2.095].

[8] W. Kim, T. H. Kim, J. Choi and H. Y. Kim, Appl. Phys. Lett. 94, 1908 (2009) [DOI: http://dx.doi.org/10.1063/1.3089820].

[9] W. Giggenbach, Inorg. Chem. 10, 1333 (1971) [DOI: http:// dx.doi.org/10.1021/ic50101a002].

[10] M. Lucas,A. Gachagan and A. Cardoni, Journal of Mechanical Engineering Science. 223, 2949 (2009) [DOI: http://dx.doi. org/10.1243/09544062JMES1671].

[11] Ministry of Food and Drug Safety: Korean Pharmaceutical Codex(3rd edition, 2007) p.1271.

[12] G. K. Stookey, T. A. Burkhard and B. R. Schernehorn, J. Dent. Res. 61, 1236 (1982) [DOI: http://dx.doi.org/10.1177/0022034582061 0110501]. 